\title{
Migrantes bolivianos/as y luchas por el derecho a la ciudad en Argentina. Una revisión crítica de la perspectiva de la Autonomía de las Migraciones
}

\section{Bolivian migrants and struggles for the right to the city in Argentina. A critical review of the perspective of the Autonomy of Migration}

Federico Rodrigo ${ }^{a *}$ (I) https://orcid.org/0000-0002-7874-3432
Recibido el 27 de noviembre de 2020. Aceptado el 12 de julio de 2021. Publicado el 19 de julio de 2021.

Autor para correspondencia: Federico Rodrigo. Correo electrónico: federodrigo@gmail.com

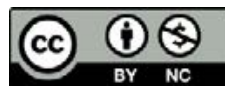

Esta obra está protegida bajo una Licencia Creative Commons Atribución-NoComercial 4.0 Internacional.

\begin{abstract}
${ }^{a}$ CONICET, Instituto de Desarrollo Económico y Social, Centro de Investigaciones Sociales, Argentina, correo electrónico: federodrigo@gmail.com
\end{abstract}

\section{Resumen}

En este artículo se desarrolla un abordaje de las disputas en torno al "derecho a la ciudad" que en los últimos años protagonizan bolivianos/as asentados/as en La Plata, en el Área Metropolitana de Buenos Aires en la Argentina. A partir de analizar sus características y de reconocer las condiciones sociales y políticas que las posibilitaron, se presenta una reflexión crítica sobre la conceptualización de las "luchas migrantes" desarrollada por algunos/as exponentes de la Autonomía de las Migraciones buscando ampliar y complejizar su lectura. En contraposición a lo planteado por esta perspectiva, concluimos que los conflictos estudiados no se emprenden contra el régimen de nacionalidad-ciudadanía cuestionando su existencia y que se desarrollan imbricados con los mecanismos y técnicas de control estatales. Es decir, cuando los/as migrantes pueden acceder a la regularidad y a documentación en sus contextos de asentamiento, se valen de los capitales y posiciones que les habilita el marco institucional para disputar el lugar de los extranjeros/as en la nación y ampliar sus derechos y/o su efectivización.

Palabras clave: migraciones, ciudadanía, nación, ciudad, luchas.

\begin{abstract}
In this article we develop an approach to the disputes around the "right to the city" that Bolivians have settled in La Plata in recent years, in the Metropolitan Area of Buenos Aires in Argentina. Based on analyzing their characteristics and recognizing the social and political conditions that made them possible, we present a critical reflection on the conceptualization of the "migrant struggles" developed by some exponents of the Autonomy of Migration, seeking to broaden their reading. We conclude that the conflicts studied are not undertaken against the nationality-citizenship regime, questioning its existence and that they
\end{abstract}


develop imbricated with the state control mechanisms and techniques. That is, when migrants can access regularity and documentation in their settlement contexts, they use the capitals and positions that the institutional framework enables them to dispute the place of foreigners in the nation and expand their rights, and/or its effectiveness.

Keywords: migrations, citizenship, nation, city, struggles.

\section{Introducción}

En los últimos años, en un contexto de aumento de las restricciones y predominio de un enfoque securitario sobre la movilidad humana que fue caracterizado como un "sistema global de fronteras" (Varela Huerta, 2015; Domenech, 2020), distintos/ as autores/as inspirados/as principalmente en la perspectiva de la Autonomía de las Migraciones pusieron el foco sobre las "luchas migrantes". Así fueron definidas las "luchas medianamente organizadas en las cuales los/as migrantes desafían, derrotan, evaden o dificultan las políticas de movilidad dominantes (incluyendo el control fronterizo, la detención y la deportación) o el régimen de trabajo, o el espacio de ciudadanía" (De Génova et al., 2015, p. 80).

Diferentes investigaciones buscaron indagar las formas de disidencia que se oponen a la "extranjerización permanente" (Varela Huerta, 2015) que operan los regímenes de los Estados-nación sobre ciertas personas y que no son perceptibles desde, y no encajan en, los paradigmas establecidos de representación política. Estas conceptualizaciones se nutrieron principalmente del análisis de movilizaciones y reclamos por derechos protagonizadas por sujetos no considerados ciudadanos de acuerdo con los marcos institucionales. Entonces, a través de sus performances de protesta, "migrantes sin papeles" y otros grupos excluidos cuestionan los modos de establecimiento de los límites de la comunidad política (Balibar, 2012; Mezzadra \& Neilson, 2016; Cordero et al., 2019; Casas-Cortes \& Cobarrubias, 2020).

La ampliación de las posibilidades analíticas y de la imaginación política que conlleva esta perspectiva, sin embargo, presenta ciertas limitaciones en su valoración de algunos de los aspectos más relevantes de numerosos conflictos protagonizados por migrantes. Si el foco se sitúa fundamentalmente en torno a las tensiones que los/ as extranjeros/as introducen en el régimen de control migratorio y de exclusión por medio de la articulación entre nacionalidad y ciudadanía, ¿no se descuidan aspectos fundamentales de las demandas que no se articulan en torno a este eje?

Esta interrogante es especialmente relevante en contextos como el argentino, en el cual si bien con déficits y lamentables excepciones (Caggiano, 2008; Cerrutti, 2010; Domenech, 2020), la mayoría de los/as migrantes que arriban provenientes de los países del Mercosur se valen de las posibilidades que les otorga - y que coproducen mediante sus luchas- la "hospitalidad selectiva" (Domenech, 2020) y pueden 
acceder a la tramitación de la residencia e, inclusive, a la doble nacionalidad. ${ }^{1}$ De esta manera, el acceso a la regularidad administrativa otorga ciertas particularidades a sus modos de experimentar y disputar su participación en la comunidad política. ¿Cómo conceptualizar los reclamos que se desarrollan desde posiciones étnico-nacionales (en nombre de bolivianos/as, paraguayos/as, peruanos/as, etc.) por trabajo, producción, discriminación, acceso a tierras, seguridad, etc.?

A partir de abordar luchas en torno al "derecho a la ciudad" que protagonizan migrantes bolivianos/as asentados/as en el Área Metropolitana de Buenos Aires específicamente en la ciudad de La Plata ${ }^{2}$ - en la Argentina, y las condiciones sociales y políticas que las posibilitaron, consideramos necesario revisar los modos en los que la propuesta teórica de la Autonomía de la Migraciones concibe las luchas migrantes. Especialmente en los últimos años, la extranjería emerge como una dimensión que articula en parte las dinámicas de organización y acción colectiva en villas y asentamientos informales de las grandes y medianas ciudades del país, incidiendo en la configuración de demandas y en las lógicas que adquieren los conflictos (Cravino, 2006; Baeza, 2014; Canelo, 2016; Perissinotti, 2016; Vaccotti, 2017; Magliano \& Perissinotti, 2020). En diálogo con un amplio campo de estudios que aborda la articulación entre migraciones, problemas habitacionales y producción social del hábitat en la Argentina contemporánea, buscamos iluminar disputas que exceden la problemática de la situación jurídica de sus protagonistas y que se articulan en torno al acceso a la vivienda (Magliano et al., 2015; Perissinotti, 2016; Vaccotti, 2017) y, en términos más generales, a lo que Harvey (2008) llamó el derecho a la ciudad. Es decir, que apuntan a la producción del espacio urbano, sus usos y ocupantes: vinculadas al acceso a tierra, a la vivienda, al equipamiento urbano y al uso del espacio público.

Nuestro objetivo es iluminar un tipo de conflictos de suma relevancia cuyas principales características quedan desplazadas por los lineamientos de la Autonomía de las Migraciones y que no se asimilan a las principales referencias empíricas que sus autores/as refieren en su formulación. El principal argumento de este texto es que mientras este enfoque prioriza las manifestaciones contra el régimen de nacionalidadciudadanía cuestionando su existencia, es necesario reconocer otros conflictos que se emprenden por él, buscando transformar el lugar social de los/as extranjeros/as en los contextos de recepción, ampliando sus derechos y/o su efectivización. En estas luchas el horizonte utópico no es la abolición del régimen global de fronteras, sino el fortalecimiento del lugar de ciertos colectivos en la nación de destino. Además, al menos en el caso argentino, se constituyen en la intersección entre las lógicas "territoriales" que adquirió la política en los sectores populares urbanos en las últimas

\footnotetext{
${ }^{1}$ Los principales grupos migratorios en la Argentina provienen de Paraguay (30\%), Bolivia (19\%), Chile $(10 \%)$ y Perú $(9 \%)$ de acuerdo con el censo de 2010 (INDEC, 2010) y, en los últimos años, se destaca el arribo de personas desde Venezuela que se estima ya superarían a las chilenas. Como se verá en el desarrollo del trabajo luego de la sanción de la Ley № 25.871 en 2003, si bien con ciertas dificultades (Domenech, 2020) y con un lapso de discursividad oficial claramente anti migrante y de aumento de las políticas de control entre 2016 y 2019 (Canelo et al., 2018), estos grupos pudieron acceder paulatina pero masivamente a la regularidad administrativa y la documentación. Una situación completamente diferente es la de los grupos migratorios extra-Mercosur, en la que se destacan los padecimientos y persecuciones que sufren los migrantes senegaleses.

${ }^{2}$ La Plata es la capital de la principal provincia del país: la Provincia de Buenos Aires. Cuenta con una población de 740000 habitantes (INDEC, 2010) y una historia migratoria que se remonta a su fundación, a finales del siglo XIX. Actualmente, más del $5 \%$ de su población es de origen extranjero, destacándose las colectividades paraguaya, boliviana y peruana como las más numerosas.
} 
décadas (Merklen, 2005) y las políticas migratorias inclusivas de la primera década y media del siglo XXI. De esta manera, comprender las diferentes configuraciones que adquieren los conflictos que involucran a los/as migrantes nos permite visualizar los alcances y limitaciones de nuestras diferentes herramientas analíticas y de nuestra imaginación política.

Un punto muy relevante de nuestro planteo es que la sanción de la Ley $\mathrm{N}^{\circ}$ 25.871 en 2003 en Argentina — que considera a la migración como un derecho- y la implementación de programas de regularización entre 2006 y 2015 configuraron tramas políticas que permitieron la producción de nuevas demandas y posicionamientos ciudadanos de los/as migrantes. Los/as bolivianos/as, así, se constituyeron en actores relevantes y, desde el reconocimiento de su importancia social, cultural e, inclusive, electoral, reclaman mejoras en sus barrios y su integración plena en (y el acceso a los bienes que consideran propios de) la ciudad. En definitiva, en lugar de oponerse a las regulaciones migratorias que establecen límites nacionales al ejercicio de la ciudadanía, se valen de ellas para disputar su lugar en la comunidad.

\section{Organización del texto y estrategia metodológica}

El desarrollo se divide en tres secciones y, posteriormente, se exponen las conclusiones. La primera de ellas presenta una discusión conceptual con la perspectiva de la Autonomía de las Migraciones y su problematización de las "luchas migrantes", intentando sintetizar sus aportes y señalar sus aspectos problemáticos.

Luego, en el apartado "Territorialización de la política migratoria" analizamos la ampliación del reconocimiento que obtuvieron los/as migrantes y su integración en tramas fundamentales en la vida política de distintas localidades a partir de la sanción de la Ley $\mathrm{N}^{\circ} 25.871$ y de la implementación de programas territoriales de documentación. Para su confección recurrimos a los aportes de la bibliografía especializada, al análisis de documentos públicos y a entrevistas con altos funcionarios/as de la entonces Subsecretaría de Entidades y Colectividades de La Plata desarrolladas en 2015. Si bien no se recuperan de modo directo, algunos pasajes también incorporan información obtenida en entrevistas y observaciones participantes desarrolladas con integrantes de seis asociaciones de migrantes bolivianos/as de la ciudad, que desarrollamos en el marco de investigaciones previas desde 2010 hasta la actualidad (Rodrigo, 2019a, 2019b, 2021a, 2021b). ${ }^{3}$

El tercer apartado, "Experiencia urbana y acceso a la documentación: luchas por el derecho a la ciudad", fue elaborado a través de materiales etnográficos que nos permiten problematizar las demandas urbanas que los/as bolivianos/as desarrollan en un barrio de la periferia de esta localidad. El texto se basa en distintas experiencias de campo en el barrio Futuro, en la periferia de La Plata: la primera de ellas se realizó en el año 2014 y constó de la realización de entrevistas a integrantes de una Junta Vecinal de migrantes conformada para enfrentar los conflictos en torno a la titularidad de

\footnotetext{
${ }^{3}$ Las organizaciones con las que trabajamos son: el Centro de Estudiantes y Residentes Bolivianos situado en el casco urbano; la Junta Vecinal de Barrio Futuro, en la Delegación Melchor Romero; la Asociación Comunidad Plurinacional, en la Delegación Altos de San Lorenzo; la Cooperativa Moto Méndez, en la Delegación Lisandro Olmos; la Asociación de Residentes Bolivianos, también en Barrio Futuro; y el Centro Cultural Boliviano, en la Delegación Tolosa.
} 
las tierras que hay allí. Además, desarrollamos observaciones participantes en las festividades de la Virgen de Urkupiña y de Tata Toco que tiene lugar en el barrio y entrevistas con sus organizadores en 2016 y 2017. Finalmente, durante 2019 realizamos entrevistas y observaciones participantes en dos movilizaciones desarrolladas en el barrio en las que indagamos en sus experiencias y reclamos en torno al "derecho a la ciudad" (Harvey, 2008; Agier, 2015).

\section{Luchas contra y por el régimen de ciudadanía}

En los últimos años, comenzó a desarrollarse una nueva mirada y sensibilidad epistémica en torno a las migraciones (De Génova et al., 2015). En un contexto de aumento de las restricciones y predominio de un enfoque securitario en el norte global, diferentes investigadores/as problematizaron las tensiones que se producen entre las estrategias y mecanismos de gobierno de la movilidad humana y los múltiples modos de desafío a los regímenes de control que realizan las personas y los grupos (Papadopoulos et al., 2008; Mezzadra, 2012; Mezzadra \& Neilson, 2016; Cordero et al., 2019).

En este marco de preocupaciones, hacia finales del siglo XX e inicios del XXI se conformó la denominada perspectiva de la Autonomía de las Migraciones, que buscó restituir la comprensión de las nuevas formas de subjetividad y agencia política migrante, definiendo como horizonte de reflexión a los dispositivos, experiencias y conflictos que se constituyen alrededor de la inscripción de la fuerza de trabajo en las relaciones de producción capitalistas (Cordero Díaz \& Cabrera García, 2016; Cordero et al., 2019; Casas-Cortés \& Cobarrubias, 2020). En términos teóricos esto implicó un distanciamiento de las explicaciones que entienden al movimiento de personas como un efecto de condiciones estructurales o de la racionalidad económica de los sujetos para caracterizarlo como un "exceso" que no puede ser reducido a los códigos del Estado y el capital (Mezzadra, 2012), lo que lo instituye como un acto político en sí mismo (Mitropoulos, 2011).

Esta perspectiva amplia, heterogénea y en desarrollo tiene como uno de sus aspectos fundamentales reconocer a la migración como una fuerza constituyente que precede y transforma a las formas de producción y sujeción política (Papadopoulos et al., 2008). En este sentido, sus exponentes llaman a entender a las técnicas y tecnologías de control como mecanismos que se proponen capturar las prácticas de "fuga" y de "desobediencia" a los diferentes tipos de fronteras que instauran los dispositivos dominantes (Cordero et al., 2019). Y, al mismo tiempo, señalan que las luchas de los/as migrantes emergen en contra de estos dispositivos, llevando a movilidades que exceden esos regímenes.

Algunos de los exponentes de esta perspectiva problematizaron la conceptualización de las fronteras como "muros" cuya única funcionalidad es la de excluir a ciertos grupos de personas y señalaron que la regulación de la movilidad humana ha sido un aspecto inescindible del desarrollo del capital (De Génova, 2002; Mezzadra, 2012; Mezzadra \& Neilson, 2016). Es decir que "no hay capitalismo sin migración, y que el régimen que intenta controlar o atenuar la movilidad de la mano de obra juega un papel estratégico en la constitución del capitalismo y las relaciones de clase" (Mezzadra, 2012, p. 164). Desde las políticas aperturistas — que amplían la competencia en el mercado laboral- hasta las que persiguen a los/as migrantes — situándolos en condiciones 
de vulnerabilidad que permiten su contratación por sueldos muy por debajo de la media-, el capital desarrolla formas de explotación de (y a través del) movimiento de las personas. En este sentido, propusieron que los mecanismos de control no se orientan a la exclusión de los/as extranjeros/as, sino a medir y administrar la movilidad en términos económicos. De esta manera, la regularización tanto como la ilegalización serían procedimientos de "inclusión diferencial" (Mezzadra \& Nielson, 2016) que buscarían garantizar el bajo costo de la fuerza de trabajo (De Génova, 2002). Por este motivo, afirman que el régimen de ciudadanía opera como mecanismo de asignación de los trabajadores en una jerarquía de estatus.

Por otro lado, esta perspectiva plantea que a pesar de estar inscriptas en las relaciones de poder que configuran el Estado y el capital, las prácticas y luchas de los/ as migrantes no se encuentran totalmente subsumidas a sus parámetros. Al enfatizar su "autonomía", buscaron poner sobre relieve luchas que no son perceptibles desde los criterios de visibilidad y representación política establecidos. En este sentido, proponen que las "luchas migrantes" —en las cuales actores que no son formalmente reconocidos se posicionan como sujetos de derecho, rechazando el estatus que les atribuye el Estado- ponen en crisis los mecanismos excluyentes que fundamentan el orden nacional y la soberanía estatal (Balibar, 2012; Papadopoulos \& Tsianos, 2013; Caraus, 2018). De este modo, observan que quienes las protagonizan no buscan integrarse dentro de un marco legal y político ya existente, sino que el aspecto más relevante de su intervención es el cuestionamiento del concepto de "extranjería" y su oposición a los modos hegemónicos de gestión del movimiento poblacional (Varela Huerta, 2015). De esta manera, al impugnar la lógica que organiza la pertenencia a la comunidad política y cuestionar su articulación con la nacionalidad, las "luchas migrantes" son, también, manifestaciones contra algunos de los principios rectores del capitalismo contemporáneo.

A su vez, algunos trabajos pusieron el foco en la cotidianidad y señalaron que el antagonismo que encarnan los/as migrantes se debe entender también desde la dimensión de la reproducción social de la vida. Así señalaron que las practicas individuales y colectivas con las que en diferentes contextos de recepción burlan los cercos y obstáculos impuestos deben ser consideradas parte de sus luchas (Cordero Díaz \& Cabrera García, 2016).

En la Argentina, algunos grupos de migrantes extra Mercosur - como los/ as provenientes de Senegal- que son objeto de diferentes tipos de persecuciones, están protagonizando en los últimos años algunas protestas y reivindicaciones que se asemejan a las luchas migrantes que analizan estos/as autores/as. Del mismo modo, especialmente entre 2017 y 2019, se conformaron colectivos y alianzas que buscaron rechazar el giro restrictivo de la política migratoria desarrollada por el gobierno del presidente Mauricio Macri, entre los cuales se dio un cuestionamiento a la articulación entre nacionalidad y ciudadanía y se recuperaron consignas de movimientos situados en el norte global (Gavazzo, 2018; Rho, 2020).

Sin embargo, a partir de la sanción de la Ley № 25.871 que reconoció a la migración como un derecho y brindó posibilidades de regularización especialmente de los/as migrantes de los países miembros del Mercosur, solo una pequeña porción de los conflictos que tienen a los/as extranjeros/as como actores protagónicos se desarrollan bajo las lógicas que destacan los/las autores/as de la Autonomía de las Migraciones. En la medida en que el objeto de estas disputas son las políticas de movilidad, las acciones que emprenden quienes ya han accedido a una condición administrativa regular y 
demandan el cumplimiento y/o la ampliación de los derechos que los ligan a sus conciudadanos/as quedan fuera de la definición. ¿Qué ocurre con los/as migrantes que reclaman la titularidad de las tierras en sus espacios de asentamiento, que giran en torno a reivindicaciones laborales o productivas o que demandan la habilitación de ciertas prácticas (como festividades nacionales o religiosas) en determinados espacios públicos de las ciudades? ¿No tensionan las posiciones del campo político y los procedimientos institucionales? ¿Su condición migrante es subsumida y/o asimilada en y por el marco de ciudadanía nacional de sus contextos de recepción?

Nuestro punto de partida es asumir que la ciudadanía no implica una condición estática, asociada con el estatus legal y con prácticas y posiciones institucionalmente predefinidas. Por el contrario, la entendemos como un proceso "conflictiv[o] vinculad[o] al poder, que refleja las luchas acerca de quiénes podrán decir qué, al definir cuáles son los problemas comunes y cómo serán abordados" (Jelin, 1993, p. 25). Entonces, sin desconocer los aportes de la perspectiva citada, consideramos necesario atender a otra clase de conflictos en los cuales los/as migrantes disputan su lugar y reconocimiento en el marco institucional buscando garantizar y expandir sus derechos. Es decir, no luchas contra sino por el régimen de ciudadanía.

En estas luchas por el régimen de ciudadanía se destacan algunos componentes fundamentales. Se constituyen por medio de la imbricación entre lógicas de la práctica establecidas institucionalmente y otras que desarrollan los sujetos subalternos. Es decir, como fue expuesto por diferentes autores/as, las luchas por la ciudadanía tienen lugar en el marco de, pero no se agotan en, los parámetros que definen los Estados (Isin, 2009; Acevedo Rodrigo \& López Caballero, 2012; Lazar, 2008). Por este motivo, es necesario considerar los procesos de subjetivación que tienen lugar a partir de los posicionamientos ciudadanos, que implican el entrelazamiento de categorías institucionales y los propios bagajes y experiencias de las personas y colectivos (Ong, 2012; Mitra, 2013).

Los/as autores/as de la Autonomía de las Migraciones cuestionan el punto de vista que enfatiza la capacidad de los poderes establecidos como únicos factores que definen los procesos sociales. Pero descuidan que las prácticas de resistencia también emergen inscriptas en los mecanismos de control, se constituyen tanto a partir de, como en oposición a, las categorías y posiciones de sujeto definidas por los sistemas de clasificación dominantes. En este sentido, resultan pertinentes las críticas de Nyers (2015) al modo reduccionista en que algunos/as exponentes de la mencionada perspectiva oponen los mecanismos de regulación de la población y a las prácticas migrantes, y llaman a considerar la coconstitución entre "control" y "resistencia" (Nyers, 2015). En síntesis, en lugar de partir de una oposición tajante y reificada, recuperamos los enfoques que proponen reconstruir los espacios relacionales en los que se conforman los dispositivos de poder tanto como la agencia de los sujetos subalternos.

$\mathrm{Al}$ mismo tiempo, la regularidad administrativa e, inclusive, el acceso a documentación, no conllevan en sí los atributos necesarios para el reconocimiento de la legitimidad de los sujetos que los portan, sino que las posibilidades de movilizar demandas en nombre de un colectivo ético-nacional se constituye y reproduce contingentemente en escenarios políticos concretos. Es decir, la fortaleza que brindan los documentos en diferentes conflictos no se encuentra contenida en ellos, sino que resulta en parte de la capacidad de apropiación de quienes los portan. Así, el reconocimiento que instituciones estatales y organizaciones sociales y políticas brindan a los colectivos de migrantes forma parte de lo que está en juego en sus disputas.

Por otro lado, si entendemos que los Estados construyen marcos interpretativos nacionales dentro de los cuales adquieren significado las diferentes categorías sociales 
regulando los modos de ser otro en la nación (Segato, 2007; Briones, 2005), delimitando las posibilidades de adscripción identitarias de, por ejemplo, los grupos migratorios, las luchas que buscamos destacar ponen en tensión las posibilidades de intervención y reconocimiento público desde posiciones de extranjería. En la Argentina, la hipervisibilización discriminatoria y la conformación de una etnicidad defensiva como respuesta en la década de 1990, la subsunción de las posiciones particularistas en la viabilidad de un "proyecto nacional" en el marco de la crisis de 2001-2002 (Grimson, 2006, 2009) y la emergencia reciente de nuevas demandas y protagonismos de los colectivos de migrantes (Courtis \& Penchaszadeh, 2015; Magliano et al., 2015; Vaccotti, 2018; Penchaszadeh \& Condori Mamani, 2017) constituyen algunas configuraciones contextuales a partir de, y sobre las que, intervienen los colectivos.

En las últimas décadas en numerosos países del mundo se desarrollaron fuertes transformaciones legales que incidieron en la relación formal entre nacionalidad y ciudadanía (Sassen, 2002; Caggiano, 2018). Sin ánimo de sintetizar los extensos debates que han surgido en el ámbito académico al respecto, queremos destacar que, si bien ambas cuestiones se encuentran estrechamente ligadas, su articulación concreta resulta de configuraciones jurídicas y socioculturales contingentes. Es decir, ser ciudadano/a y ser nacional, así como los regímenes que delimitan el acceso a estas condiciones, no representan equivalencias absolutas, lo que posibilita luchar por derechos ciudadanos desde posiciones de extranjería o buscar ampliar el reconocimiento social como extranjeros/as desde posiciones ciudadanas.

Entonces, tenemos dos conceptualizaciones diferentes. Por un lado, las "luchas migrantes" cuyo objeto son las políticas de control y que, a través de su impugnación, cuestionan el régimen de ciudadanía ligado a la nacionalidad y minan un parámetro fundamental de la gestión de la mano de obra en el modo de producción capitalista. Por el otro, las luchas cuyo objeto es el lugar de los/as migrantes en el régimen de ciudadanía y, a partir de ello, ponen en cuestión las lógicas, parámetros y límites de este. Entendemos que atender a las configuraciones que adquieren los conflictos puede ayudarnos a visualizar los alcances y limitaciones de nuestras herramientas analíticas.

En el caso argentino, las políticas migratorias fueron un factor fundamental del desarrollo de luchas migrantes por el régimen de ciudadanía. Pero no lo fueron por convertirse en objeto de las disputas, sino porque en torno a su implementación y al modo en el que se entramaron con otras dinámicas sociales y políticas preexistentes se conformaron modos novedosos de la acción colectiva de las organizaciones de personas extranjeras. Para profundizar este análisis comenzamos con reponer la conexión entre los programas de documentación desarrollados en el período 20062015 y las dinámicas de territorialización de la política en los sectores populares que venía teniendo lugar en la periferia de las grandes y medianas ciudades del país desde fines del siglo XX.

\section{Territorialización de la política migratoria}

En un contexto de aumento de la exclusión y de desarticulación de los actores sindicales como el que caracterizó a la Argentina fundamentalmente a partir de la década de 1980, tuvo lugar un proceso conjunto de transformación de las políticas sociales y las modalidades de organización y movilización de los sectores populares 
urbanos. Los programas de asistencia alimentaria o que brindan un ingreso mensual en contraprestación de actividades productivas, comunitarias y de formación concebidos para enfrentar la creciente pobreza y conflictividad social del periodo tuvieron un fuerte impulso. Estas políticas fueron coconformadas por movimientos y referentes que se constituyeron en los barrios de las periferias empobrecidas: primero en torno a la demanda y distribución de alimentos y, luego, para movilizar los reclamos de un nuevo actor social, los "trabajadores desocupados". Especialmente a partir de 2002, a través de diferentes modalidades de protesta y negociación comenzaron a acceder a cupos de estos programas que ejecutan por medio de la conformación de cooperativas de trabajo (fundamentalmente de construcción o limpieza) que realizan tareas encomendadas por distintos organismos estatales (Svampa \& Pereyra, 2003; Cerrutti \& Grimson, 2004; Merklen, 2005; Ferraudi Curto, 2009).

De esta manera, la desafiliación del mundo laboral formal y el distanciamiento del entramado institucional que garantizaba la satisfacción de los derechos sociales habría sido compensado en parte mediante un progresivo "repliegue hacia el barrio" (Merklen, 2005). Allí se constituyeron mecanismos de asistencia, emprendimientos laborales articulados con las políticas sociales y ganaron visibilidad los colectivos que demandan y coproducen estos dispositivos (Manzano, 2007).

Por su parte, en la medida en que muchas de las periferias urbanas donde se consolidaron estas modalidades son también zonas de asentamiento de migrantes, esta dinámica —que Merklen (2005) caracterizó como la "inscripción territorial" de la política en los sectores populares- comenzó a formar parte de sus procesos de incorporación (Glick Schiller et al., 2006). En primer lugar, los programas sociales y las organizaciones territoriales que los demandan y gestionan comenzaron a ser visualizados como una vía de cumplimento de diferentes derechos. De esta manera, especialmente a partir de la segunda mitad de la década de 2000, en diferentes zonas los/as migrantes se volvieron integrantes de suma relevancia (cuantitativa y cualitativa) de las mismas (Vázquez, 2005; Dodaro \& Vázquez, 2008; Halpern, 2009; Grimson, 2009; Rodrigo, 2018, 2019c). A su vez, este reconocimiento se convirtió en un importante incentivo para el acceso a la regularidad administrativa y la documentación, que constituían requisitos formales para su tramitación.

En 2003 se sancionó la Ley $\mathrm{N}^{\circ} 25.871$ que reconoció a los/as migrantes como "sujetos de derecho" abriendo un escenario novedoso al recuperar un universo de sentidos que articula derechos humanos, políticas de la diferencia y el desarrollo de procesos de integración regionales (Domenech, 2007; Vichich, 2005; Novick, 2011). A su vez, una de las principales características de la política migratoria emprendida entre 2003 y 2015 fue el desarrollo de programas de regularización. Se destacan en este sentido el Programa Patria Grande (2006-2010) y el Programa Territorial de Acceso a la Documentación (2013-2015), que apuntaron a garantizar la regularización de la situación administrativa de los/as residentes extranjeros/as. En este marco, se generaron condiciones que redefinieron tanto su relación con las instituciones estatales como sus lógicas de intervención en el espacio público: podemos denominar estas transformaciones como una territorialización de la política migratoria.

Domenech (2009) caracterizó la participación de movimientos y organizaciones de derechos humanos en la determinación de los asuntos ligados a la movilidad hacia fines de la década de 1990 y el desplazamiento de la perspectiva que en Argentina tuvo lugar a partir del cambio de la ley como una "ciudadanización de la política migratoria”. Es decir, que los marcos interpretativos, lógicas de acción, demandas y 
medidas estatales se elaboraron en torno al lenguaje de derechos. Los programas de documentación desarrollados a partir de 2006, por su parte, ampliaron y redefinieron esta lógica.

Estos programas implicaron la realización de "operativos territoriales" y una "descentralización operativa" ejecutada en articulación con los gobiernos provinciales y municipales. A su vez, se habilitó la participación de asociaciones civiles en la tramitación de las solitudes de residencia y nacionalidad bajo la figura de Instituciones Sociales Colaboradoras. Los operativos se realizaban a través del desplazamiento del personal de la Dirección Nacional de Migraciones (muchas veces con integrantes de otras áreas del Ministerio del Interior de la Nación) y de los municipios a distintos barrios, para, una vez instalados allí, recibir a los/as habitantes de la zona para que realizaran sus trámites de regularización. En términos prácticos, este tipo de procedimientos suponía, también, el trabajo de organizaciones locales para que se garantizara la difusión de la actividad y el acercamiento de un número considerable de personas.

Así, los programas integraron una cantidad relevante de actores que incidieron en las características y la dinámica que adoptaron en las distintas localidades donde fueron ejecutados. De esta manera, los documentos y los mecanismos para acceder a ellos fueron incorporados en las lógicas de relación de los actores que componen a los barrios como espacios políticos y, al mismo tiempo, viabilizaron la construcción de relaciones entre funcionarios/as estatales y referentes de distintos colectivos de migrantes.

Por otro lado, especialmente en ciertos municipios en los que los partidos gobernantes desarrollaron una estrategia al respecto, estas políticas permitieron también una ampliación muy relevante de los padrones electorales de extranjeros/ as. Su crecimiento exponencial —que, en La Plata, por ejemplo, llevó de 8800 empadronados/as en la elección de 2005 a 37000 en la de 2015- fue objeto de diferentes denuncias mediáticas que atribuían una lógica "clientelar" ${ }^{4}$ a las medidas de regularización. En esta interpretación, los/as extranjeros/as serían sujetos vulnerables y pasivos/as dispuestos a participar del intercambio de "favores" — como el acceso a un cupo en un plan social- "por votos" (Auyero, 2004). Sin embargo, sin desconocer que la motivación proselitista fue un aspecto presente en las vinculaciones que se establecieron durante la implementación del programa, aquí queremos destacar que la atribución de un capital electoral a los/as migrantes no solo no contribuyó a su vulnerabilidad, sino que, muy rápidamente, situó a sus organizaciones y referentes en una posición de reconocimiento y relevancia. Inclusive, tanto en La Plata como en otras localidades de la provincia de Buenos Aires - como Escobar para el caso de los/ as bolivianos/as-, distintos actores les comenzaron a atribuir la victoria o la derrota de un/a determinado/a candidato/a.

En síntesis, los programas de documentación "territoriales" y las dinámicas políticas que se articularon en torno a su ejecución redefinieron el lugar de los/as migrantes en espacios sociales concretos, volviéndolos/as sujetos relevantes en algunas de las dinámicas de los barrios populares. Si bien en la Argentina en el año 2016 comenzó a implementarse una política mucho más restrictiva y, a partir del Decreto $\mathrm{N}^{\circ} 70 / 2017$, se limitaron muchos de los aspectos progresivos de la Ley $\mathrm{N}^{\circ} 25.871$ (Canelo et al., 2018), las transformaciones en las agendas de las organizaciones de migrantes y en

${ }^{4}$ Para una caracterización y análisis en profundidad de este fenómeno, ver Auyero (2004) y Vommaro y Quirós (2011). 
el reconocimiento de las instituciones estatales y civiles argentinas evidencian que los procesos de movilización y posicionamiento ciudadano tramados en el periodo anterior no se han detenido.

\section{Experiencia urbana y acceso a la documentación: luchas por el derecho a la ciudad}

La nueva visibilidad que adquirieron los/as migrantes también formó parte del desarrollo de lógicas de movilización —y, en términos más generales, de subjetivación política y ciudadana - en cierta medida novedosas. Los/as a migrantes bolivianos/as en el Área Metropolitana de Buenos Aires, especialmente a partir de la segunda mitad de la década de 2000, además de fiestas religiosas, peñas, grupos de baile, campeonatos de futbol, celebraciones "originarias" o programas radiales que constituyen lo que podemos denominar la "agenda clásica" de sus entidades (Grimson, 1999; Gavazzo, 2004; Giorgis, 2004; orm-CeMla, 2004; Caggiano, 2005; Canelo, 2013), comenzaron a desarrollar otras demandas ligadas a la inseguridad y el acceso al suelo urbano.

En las últimas décadas en muchas ciudades argentinas se desarrolló un proceso sostenido de incremento del precio del suelo (Cisterna \& Matteucci, 2015). Esta dinámica de mercado incidió directamente en las restricciones ya existentes al acceso a la vivienda propia en los sectores populares, dificultando su concreción por la vía comercial. En estas condiciones, las tomas colectivas informales de tierra se sucedieron como parte de la estrategia de diferentes organizaciones sociales y políticas. Al mismo tiempo, ante la necesidad creciente de tierra por la ampliación demográfica y la falta de políticas de producción y regulación de suelo urbano también se conformaron loteos comerciales de modo informal. Ambas dinámicas dieron origen a numerosos asentamientos en los que la titularidad de las parcelas es objeto de disputa entre vecinos/as, organizaciones sociales, emprendimientos privados e instituciones estatales. En este marco, sus habitantes adoptaron diferentes estrategias para solicitar la integración de estos barrios a las políticas urbanas y el acceso a diferentes servicios (Cravino, 2006, 2014; Van Gelder et al., 2013). Por su parte, el reconocimiento de los/ as migrantes y la interlocución con sus asociaciones y referentes que se dinamizaron a partir de los programas de regularización permitió que también se sitúen como actores legítimos en los reclamos por el "derecho a la ciudad" ante las autoridades.

Los déficits habitacionales que experimentan los/as migrantes en Argentina, entre los que se destacan el hacinamiento, la precariedad de las viviendas, la irregularidad en la tenencia de los lotes y la falta de acceso a servicios básicos, han sido extensamente tratados por la bibliografía especializada (Grimson, 2009; Matossian, 2010; Caggiano \& Segura, 2014; Baeza, 2014; Mera, 2017; Magliano \& Perissinotti, 2020). Las investigaciones destacan que simultáneamente a las dificultades que padecen por su extranjería, deben soportar diferentes dinámicas de discriminación vinculadas a su zona de residencia y sufren el "efecto de lugar" (Dahau \& Giglia, 2008) sobre distintas prácticas, situando a la distribución espacial como una forma de "inclusión diferencial" (Mezzadra \& Neilson, 2016) de esta población que constituye, entre otros factores, su estatus subordinado.

$\mathrm{Al}$ mismo tiempo, especialmente en los últimos años, numerosos trabajos vienen reconociendo dinámicas de organización y acción colectiva en villas y asentamientos 
informales de las grandes y medianas ciudades del país protagonizadas por migrantes (Cravino, 2006; Baeza, 2014; Magliano et al., 2015; Canelo, 2016; Perissinotti, 2016; Vaccotti, 2017; Magliano \& Perissinotti, 2020). En ellos se desarrolla una agenda de reivindicaciones amplia, que adquiere diversas articulaciones con la extranjería. Buscando iluminar disputas en las que la regulación jurídica o el acceso a la documentación quedan desplazados a un segundo plano, Perissinotti las caracterizó como luchas por "un lugar donde vivir" (2016, p. 62).

"Futuro" es uno de los 152 asentamientos y villas consignados por el Registro Nacional de Barrios Populares ${ }^{5}$ en La Plata. Forma parte de la delegación Melchor Romero ubicada en la periferia sudoeste de la ciudad. Su ocupación inició en el año 1996 y, especialmente a partir de la década de 2000, tuvo un fuerte crecimiento demográfico hasta superar las 900 familias en la actualidad (Adriani et al., 2020). Allí también se incorporaron numerosos/as migrantes bolivianos/as provenientes de los departamentos de La Paz, Tarija, Oruro y, principalmente, Cochabamba. Si bien no existe un censo que confirme su proporción cuantitativa (que sin duda es muy elevada), constituyen el grupo identitario más visible, lo que permite que la zona sea denominada como "barrio de bolivianos/as".

Una de las características fundamentales de "Futuro", como del resto de los denominados "barrios populares" de la Argentina, es que la mayor parte de sus vecinos/ as no cuentan con título de propiedad, boleto de compraventa u otro documento que acredite la titularidad de los lotes y las viviendas en las que habitan. El desarrollo informal de esta clase de asentamientos, a su vez, también genera otras dificultades como la precariedad de las calles, la falta de recolección de basura y de luminarias, así como la imposibilidad de acceder legalmente a los servicios de luz, gas, agua corriente, etc. La problemática de la inseguridad y el delito, por su parte, también se encuentra entre las principales preocupaciones de sus habitantes, que afirman sentirse descuidados/as por las autoridades y a merced de todo tipo de peligros.

En los últimos años estas problemáticas dinamizaron la constitución de distintos núcleos organizativos y fueron el motivo de diferentes demandas y protestas llevadas adelante en el barrio. En otros trabajos dimos cuenta del surgimiento de una Junta Vecinal conformada principalmente por migrantes, que recuperaron su experiencia de organización en el área rural de Cochabamba para enfrentar las disputas — que en algunos casos llevaron a enfrentamientos violentos- por la tierra en esta zona de La Plata (Rodrigo, 2019a, 2021a). Al mismo tiempo, también se destaca la presencia de numerosos comedores comunitarios de distintas organizaciones sociales y políticas, así como espacios religiosos católicos y evangélicos que, a pesar de no estar constituidos en torno a identificaciones bolivianas/as, también operan como ámbitos de encuentro étnico-nacionales y de elaboración colectiva de las dificultades que atraviesan.

Saida, por ejemplo, es una mujer proveniente del Departamento de Potosí, que arribó a La Plata con poco más de 20 años a comienzos de la década de 2000. Su vínculo con Futuro comenzó a partir de su participación en una iglesia evangélica en la que se destaca la presencia de mujeres bolivianas, a la que asistía también una de

\footnotetext{
${ }^{5}$ El Registro Nacional de Barrios Populares fue establecido por el Decreto № 358/2017. Considera como barrio popular a las zonas de vivienda donde viven al menos a ocho familias agrupadas o contiguas, donde más de la mitad de la población no cuenta con título de propiedad del suelo ni acceso regular a por lo menos dos de los tres servicios básicos: red de agua corriente, red de energía eléctrica con medidor domiciliario y/o conexión a la red cloacal formal.
} 
sus hermanas. Luego de vivir en distintas partes de la ciudad, se mudó al barrio en donde consiguió trabajo por medio de la política social y pudo dejar su actividad como empleada doméstica. En una entrevista realizada en el invierno de 2019, nos comentó que desde antes de habitar en el lugar ya participaba de diferentes reclamos allí:

No se podía caminar. Yo venía en remis a la iglesia y [nos] tenía que dejar en $155 .{ }^{6}$ Era todo barro, no se podía entrar. Teníamos [que caminar] más de diez cuadras así y era todo campo. Y ahí las hermanas nos avisaban, que iban a cortar para pedir asfalto y dejar de estar aislados. [Los/as responsables políticos/as] discriminación tienen. Nosotros somos bolivianos pero estamos legales [regulares], no nos dan importancia. (Entrevista a Saida, 2019)

Tanto en este testimonio como en muchos otros relatos, los "papeles" aparecen como objetos protagónicos de los acontecimientos. Tener credenciales (o no tenerlas) es mencionado como un aspecto relevante en la decisión de emprender un reclamo y en las negociaciones que se suscitan. En todas estas circunstancias, las identificaciones personales son percibidas como capitales en los procesos de disputa; en este caso, en el reclamo por la pavimentación de las calles.

Saida se refiere a los cortes de la intersección de las avenidas 32 y 155 que comenzaron a realizar diferentes grupos de vecinos/as desde mediados de la década de 2000. Si bien las mujeres bolivianas de su iglesia no promovían las acciones, el grupo religioso funcionaba como un espacio de socialización en el que se abordaban las problemáticas y circulaba información referida a las protestas. Junto con ellas, entonces, se integró en las manifestaciones que buscaban facilitar el ingreso y la salida de vehículos al barrio.

Las calles emergen en diferentes relatos como un factor clave de las problemáticas barriales en la medida en que su mal estado obstaculiza múltiples actividades. Su deterioro es una parte fundamental de la concepción de relegamiento que muchos/ as vecinos/as sienten que tiene el barrio. Los/as migrantes afirman estar "aislados" u "olvidados", sin la posibilidad de contar con servicios que lleguen hasta sus casas. Los reclamos por seguridad se constituyen en este mismo universo de sentidos.

En una manifestación organizada por este motivo luego del asesinato de un joven del barrio a fines de 2019 nos encontramos con algunas personas que conocíamos de nuestras experiencias de campo anteriores y de las conversaciones con los/as integrantes de la Junta Vecinal. Daniel, un cochabambino de cerca de 40 años que trabaja en la construcción nos comentaba por qué se había sumado a la manifestación antes de seguir camino hacia su trabajo:

Todo va enlazado. Primero las luminarias, la calle que está rota, el micro que está por dejar de pasar, los distintos casos de apunalamientos, robo. Este año me desvalijaron la casa, fui a hacer la denuncia y no me tomaron. No te atienden. Te tienen esperando, dando vueltas y no hacen nada. (Entrevista a Daniel, 2019)

Otras mujeres integrantes de cooperativas de limpieza municipal que habían dejado sus tareas para participar de la manifestación reforzaban la idea de que la "discriminación al barrio" estaba determinada principalmente porque la mayoría de sus habitantes son extranjeros/as:

${ }^{6}$ La intersección entre las avenidas 32 y 155 marcan el ingreso al barrio y el final del asfalto. 
Eso es verdad. Ayer a las dos de la tarde mi hijo estaba regresando de la facultad (...). Y agarraron allá, al frente de los eucaliptos .... Vamos a la comisaría [del barrio] y yo les dije "llamamos al 911, ¿que no les pasa información?". "Ah, pero nosotros no estamos a cargo para ver ese movimiento [coordinar la intervención policial a partir de las denuncias que se reciben al 911], eso tendría que hacerse cargo de comando central”. Yo les dije: “¿por qué ustedes no dan importancia? Tu gesto dice que no te importamos, somos personas. Extranjeros, pero estamos dignamente, nos tienen que dar el derecho de la persona. No importa que seamos bolivianos, o negros o blancos". (Entrevista a Mabel, 2019)

Tanto en las movilizaciones para reclamar seguridad como en las que piden otras mejoras como la pavimentación de las calles se destaca la vinculación que los/as manifestantes realizan entre la relegación del barrio y la discriminación que sufren las personas bolivianas. Pero aquí, a diferencia de lo ocurrido hacia fines de la década de 1990 y comienzos de la de 2000, su rechazo no se circunscribe a la "reivindicación de la cultura" o las "costumbres" que traerían los/as migrantes de sus lugares de origen, intentando demostrar atributos positivos que disputen las calificaciones xenófobas (Grimson, 1999; Caggiano, 2005). A partir del acceso a documentación y de su progresivo reconocimiento social y político, el cuestionamiento a la discriminación comenzó a formar parte de la reivindicación de derechos y de la emergencia de un posicionamiento confrontativo en diferentes conflictos, llegando en situaciones a la violencia física (Canelo, 2013; Magliano et al., 2015; Vaccotti, 2018). En este caso, queda claro en los testimonios la asunción de que, en tanto vecinos/as, estas personas merecen el reconocimiento de los/as funcionarios/as estatales y el acceso a los bienes que garantizarían su derecho a la ciudad.

Diferentes autores/as han señalado la existencia de relaciones entre la experiencia del espacio y de la ciudadanía. Por un lado, existen vinculaciones entre los sistemas de categorizaciones de los diversos grupos y las distintas configuraciones urbanas que habitan y por las que transitan. Estas correlaciones llevaron a plantear la existencia de un "capital espacial" (Prévot Schapira, 2001) o de un "efecto de lugar", que refleja la posición social de los sujetos y marca su relación con la trama urbana. Por el otro, el tipo de demandas que formulan, la intensidad de sus prácticas políticas y de su reivindicación de derechos se encuentra estrechamente ligada con el tipo de nexo que los une a distintos territorios (Dahau \& Giglia, 2008).

Holston (2008) señala que desde la década de 1970 en la periferia de las ciudades brasileras las clases trabajadoras produjeron una "ciudadanía insurgente". El proceso de organización y movilización en torno a la construcción de las casas, el acceso a los títulos de propiedad y a los servicios básicos, entre otras cuestiones, transformó a la periferia en un espacio de imaginación de un futuro alternativo. De esta manera, su experiencia en estos barrios se convirtió en el contexto y la substancia de una nueva ciudadanía conformada principalmente a través de sus luchas por el "derecho a la ciudad" (Harvey, 2008; Agier, 2015). La agenda urbana, así, adquirió tanto o más protagonismo en estos procesos de ciudadanización que las reivindicaciones laborales y/o clasistas.

Así, en diferentes circunstancias las luchas por el derecho a la ciudad ocupan un lugar central en la conformación de la relación entre sujetos y Estado. En este marco, igual que como existe un debate en torno a la causalidad en la relación entre la posición 
en el espacio geográfico y en el espacio social, también la vinculación entre experiencia espacial y ciudadana abre interrogantes relevantes. Mientras Dahau y Giglia (2008) encuentran que las condiciones de la experiencia urbana (como el acceso a títulos de propiedad de la vivienda) son un factor determinante en la elaboración de otras demandas (laborales, de reconocimiento, etc.), en nuestro trabajo observamos que la capitalización ciudadana (como el acceso a DNI y el reconocimiento social y político) es un aspecto imprescindible de las luchas por la ciudad de los/as bolivianos/as.

Los/as migrantes se sienten integrantes legítimos de la comunidad y, como tales, reclaman diferentes aspectos vinculados con su "derecho a la ciudad". En su interpretación, las trabas y dificultades que se les presentan como bolivianos/as se conectan al relegamiento del barrio. Por este motivo, en sintonía con lo registrado por diferentes estudios en distintos espacios de asentamiento, asumen a la pertenencia nacional como una dimensión de las disputas de los barrios populares (Baeza, 2014; Magliano et al., 2015; Vaccotti, 2018). En definitiva, las luchas por el derecho a la ciudad son consecuencia del acceso a la ciudadanía y, al mismo tiempo, constituyen un modo de producción y ampliación de la ciudadanía de los migrantes.

Esta conexión entre luchas articuladas localmente y luchas de ciudadanía, por otra parte, no representa una novedad en la trayectoria de muchos/as migrantes. Lazar (2008) señaló que luego de que las reformas neoliberales desarrolladas en Bolivia en la década de 1990 incluyeran como política de diversidad, ciertas reivindicaciones de los pueblos indígenas (como la posibilidad de titulación colectiva de la tierra, la educación bilingüe o la Ley de Participación Popular que reconoció a los grupos y sus líderes como actores en las decisiones municipales) en diferentes comunidades, el lugar de residencia se convirtió en un espacio de construcción de modos colectivos de conformación de las subjetividades políticas y en la relación que los sujetos entablan con el Estado.

En estos modos de producción y experiencia de la ciudadanía articulados en torno al lugar de pertenencia - fundamentalmente desarrollados a partir de las Juntas Vecinales y Escolares- encuentran cierta continuidad en los espacios de asentamiento de los/as migrantes en la Argentina. En algunos casos, como en la mencionada Junta Vecinal de barrio Futuro, la vinculación es directa al recuperar la misma lógica organizativa a la que se refiere Lazar. En otras circunstancias, los nexos son indirectos, pero mantienen una conexión clara: luchar contra la relegación de un barrio, en la perspectiva de los/as migrantes, implica al mismo tiempo una disputa por su lugar y reconocimiento en la comunidad. Así, constituyen un sujeto colectivo (la comunidad en Bolivia, el barrio en Futuro) sumamente relevante en sus modos de posicionamiento ciudadano.

Ahora bien, la posibilidad de recuperar la experiencia de las relaciones entre barrio y Estado en los contextos de asentamiento se encuentra condicionada por el acceso a la regularidad administrativa, la documentación y por cierto reconocimiento social y político de sus asociaciones y referentes. De esta manera, los bienes y símbolos que las instituciones estatales configuran como fetiches de la ciudadanía (Gordillo, 2006) también forman parte de una apropiación "desde abajo" (Jelin, 1993, 1996) que los conecta con las experiencias pre migratorias. 


\section{Conclusiones}

En este artículo buscamos caracterizar diferentes experiencias políticas que no son adecuadamente comprendidas si seguimos las conceptualizaciones desarrolladas por distintos exponentes de la Autonomía de la Migración. A partir de nuestro propio trabajo de campo y de dinámicas registradas por la bibliografía especializada en la Argentina, encontramos necesario poner sobre relieve diferentes luchas que se configuran con ciertas particularidades que las vuelven relevantes en la comprensión de las relaciones entre agencia y dispositivos institucionales.

La perspectiva de la Autonomía de las Migraciones se constituye buscando poner el foco en la capacidad de crear márgenes de acción de los/as migrantes. Su planteo es que las migraciones movilizan un momento ingobernable para la lógica del Estado y del capital, que les permite a los sujetos una fuga del orden social y de la lógica de explotación de la fuerza de trabajo. Al mismo tiempo, señalan que los regímenes de control se transforman buscando regular estas prácticas emergentes. Si bien reconoce múltiples tensiones, el planteo siempre parte de una contraposición entre mecanismos de control y sujetos. No porque desconozca que el control condiciona las prácticas, sino porque entiende que la capacidad "autónoma" de los mismos comienza cuando logran escindir de los dispositivos de regulación algunos aspectos de su producción subjetiva.

Por su parte, en los casos trabajados en este artículo, la agencia se constituye en la articulación entre experiencias pre migratorias y un modo específico de inscribirse en los dispositivos del Estado (que surgen, a su vez, de la interacción con las organizaciones sociales). En este sentido, consideramos fundamental evitar una oposición tajante y reificada entre parámetros institucionales y acción subalterna, que desconoce los múltiples modos a partir de los cuales los sujetos se valen de las posiciones que les habilitan las políticas estatales para disputar su lugar en sus contextos de recepción (y, por lo tanto, algunos de los límites que establecen esas mismas políticas). La autonomía de las luchas de los/as migrantes, así, no se constituye en su distinción respecto de los dispositivos de control, sino que emerge en las torsiones que introduce en, y a partir de, los mismos (Butler \& Spivak, 2009).

Simultáneamente, esta distinción también supone un contrapunto en torno a la relación entre nacionalidad y ciudadanía. Si la perspectiva de las "luchas migrantes" la asume como de homologación plena, en nuestro trabajo encontramos más productivo atender a las lógicas de reconocimiento y de acción (formales, pero también informales con impacto en procesos institucionales) diferenciales (Sassen, 2002) que constituyen los/as migrantes en sus disputas. En definitiva, entendemos que identificar las configuraciones que adquieren los conflictos puede ayudarnos a visualizar los alcances y limitaciones de nuestras herramientas analíticas y de nuestra imaginación política.

\section{Referencias}

Acevedo Rodrigo, A. \& López Caballero, P. (2012). Ciudadanos inesperados. Espacios de formación de la ciudadanía ayer y hoy. Centro de Investigación y de Estudios Avanzados. 
Adriani, L., Santa María, J., Peiró, M. L. \& Alzugaray, L. (2020). Barrios populares del partido de La Plata. Localización y características según delegaciones municipales. Universidad Nacional de La Plata. Facultad de Humanidades y Ciencias de la Educación.

Agier, M. (2015). Do direito à cidade ao fazer cidade. O antropólogo, a margen e o centro. Maná, 21(3), 483-498. https://doi.org/10.1590/0104-93132015v21n3p483

Auyero, J. (2004). Clientelismo político. Las caras ocultas. Capital Intelectual.

Baeza, B. (2014). "Toma de tierras" y crecimiento urbano en Comodoro Rivadavia: diferenciaciones y tensiones entre migrantes limítrofes, internos y comodorenses. Párrafos Geográficos, 13(2), 76-107.

Balibar, E. (2012). Los dilemas históricos de la democracia y su relevancia contemporánea para la ciudadanía. Enrahonar. Quaderns de Filosofia, 48, 9-29.

Briones, C. (Comp.) (2008). Cartografias Argentinas. Políticas indigenistas y formaciones provinciales de alteridad. Antropofagia.

Butler, J. \& Spivak, G. (2009). ¿Quién le canta al Estado-Nación? Paidós.

Caggiano, S. (2005). Lo que no entra en el crisol. Inmigración boliviana, comunicación intercultural y procesos identitarios. Prometeo.

Caggiano, S. (2008). "Que se haga cargo su país": la cultura, los Estados y el acceso a la salud de los inmigrantes bolivianos en Jujuy. En C. García Vázquez (Comp.), Hegemonía e interculturalidad. Poblaciones originarias e inmigrantes (pp. 243-279). Prometeo.

Caggiano, S. (2018). Blood ties: migrations, state transnationalism and automatic nationality. Ethnic and Racial Studies, 41, 267-284. https://doi.org/10.1080/01419 870.2017 .1341990

Caggiano, S. \& Segura, R. (2014). Migración, fronteras y desplazamientos en la ciudad. Dinámicas de la alteridad urbana en Buenos Aires. Revista de Estudios Sociales, $48,29-42$

Canelo, B. (2013). Fronteras internas. Migración y disputas espaciales en la Ciudad de Buenos Aires. Antropofagia.

Canelo, B. (2016). Migración y políticas públicas desde el margen. Acciones y omisiones estatales en un parque de la Ciudad de Buenos Aires. Migraciones Internacionales, 8(3), 125-153.

Canelo, B., Gavazzo, N. \& Nejamkis, L. (2018). Nuevas (viejas) políticas migratorias en la Argentina del cambio. Si Somos Americanos. Revista de Estudios Transfronterizos, $18,150-182$

Caraus, T. (2018). Migrant protest as acts of cosmopolitan citizenship. Citizenship Studies, 22(8), 791-809.

Casas-Cortés, M. \& Cobarrubias, S. (2020). La autonomía de la migración: Una perspectiva alternativa sobre la movilidad humana y los controles migratorios. EMPIRIA. Revista de Metodología de Ciencias Sociales, 46, 65-92. https://doi.org/10.5944/empiria.46.2020.26967

Cerrutti, M. (2010). Salud y migración internacional: mujeres bolivianas en la Argentina. PNUD.

Cerrutti, M. \& Grimson, A. (2004). Buenos Aires, neoliberalismo y después. Cambios socioeconómicos y respuestas populares. Cuadernos del IDES, (5). Instituto de Desarrollo Económico y Social. 
Cisterna, C. \& Matteucci, L. G. (2015). La urbanización en Argentina durante el neodesarrollismo. Una lectura desde las rupturas y continuidades con el período neoliberal. Revista de Dereito da Cidade, 7(4), 1573-1599. https://doi.org/10.12957/ rdc.2015.20922

Cordero, B., Mezzadra, S. \& Varela, A. (Coords). (2019). América Latina en Movimiento. Migraciones, límites a la movilidady sus desbordamientos. Traficantes de sueños, Universidad Autónoma de la Ciudad de México.

Cordero Díaz, B. L. \& Cabrera García, A. (2016). Aproximaciones críticas a las luchas de migrantes latinoamericanos. Antagonismo, reproducción social de la vida y "excedencia" en arenas de confrontación global. Papeles de Trabajo, 32, 35-56.

Courtis, C. \& Penchaszadeh, A. P. (2015). El (im) posible ciudadano extranjero. Ciudadanía y nacionalidad en Argentina. Revista SAAP, 9(2), 375-394.

Cravino, M. C. (2006). Las villas de la ciudad: mercado e informalidad urbana. UNGS.

Cravino, M. C. (Comp.). (2014). Derecho a la ciudad y conflictos urbanos. La ocupación del Parque Indoamericano. Universidad Nacional de General Sarmiento.

Dahau, E. \& Giglia, A. (2008). Las reglas del desorden: habitar la metrópoli. Siglo XXI Editores, UAM.

De Génova, N. (2002). Migrant "Illegality" and Deportability in Everyday Life. Annual Review of Anthropology, 31, 419-447.

De Génova, N., Mezzadra, S. \& Pikles, J. (2015). New Keywords: Migration and Borders. Cultural Studies, 29(1), 55-87. https://doi.org/10.1080/09502386.2014.89 1630

Dodaro, C. A. \& Vázquez, M. G. (2008). Representaciones y resistencias sobre/en grupos migrantes. Política y visibilidad(es). En P. Alabarces \& M. G. Rodríguez (Comps.), Resistencia y mediaciones. Estudios sobre cultura popular (pp. 139-164). Paidós.

Domenech, E. (2007). Inmigración, estado y educación en Argentina: ¿ Hacia nuevas políticas de integración? Centro de Estudios Avanzados. Universidad Nacional de Córdoba.

Domenech, E. (2009). La visión estatal sobre las migraciones en la Argentina reciente. De la retórica de la exclusión a la retórica de la inclusión. En E. Domenech (Comp.), Migración y política: el Estado interrogado. Procesos actuales en Argentina y Sudamérica (pp. 21-69). Universidad Nacional de Córdoba.

Domenech, E. (2020). La "política de la hostilidad" en Argentina: detención, expulsión y rechazo en frontera. Estudios Fronterizos, 21, e057. https://doi.org/10.21670/ ref.2015057

Ferraudi Curto, M. C. (2009). "Hoy a las 2, cabildo": etnografía en una organización piquetera. En A. Grimson, M. C. Ferraudi Curto \& R. Segura (Comps.), La vida política en los barrios populares de Buenos Aires. Buenos Aires (pp. 153-177). Prometeo.

Gavazzo, N. (2004). Identidad boliviana en Buenos Aires: las políticas de integración cultural. Theomai. Estudios sobre Sociedad, Naturaleza y Desarrollo, 9, 1-17.

Gavazzo, N. (2018) . Jóvenes migrantes e hijos de inmigrantes latinoamericanos en Buenos Aires: una generación en movimiento. Confluenze. Revista di studi iberoameriani, 10(1), 131-165. https://doi.org/10.6092/issn.2036-0967/8298

Giorgis, M. (2004). La virgen prestamista. La fiesta de la Virgen de Urkupiña en el boliviano Gran Córdoba. Antropofagia. 
Glick Schiller, N., Çağlar, A. \& Guldbrandsen, T. (2006). Beyond the ethnic lens: locality, globality, and born-again incorporation. American Ethnologist, 33(4), 612-633.

Gordillo, G. (2006). En el Gran Chaco: Antropologías e historias. Prometeo.

Grimson, A. (1999). Relatos de la diferencia y la igualdad. Los bolivianos en Buenos Aires. Eudeba.

Grimson, A. (2006). Nuevas xenofobias, nuevas políticas étnicas. En A. Grimson \& E. Jelin (Comps.), Migraciones regionales hacia la Argentina. Diferencia, desigualdades y derechos (pp. 69-97). Prometeo.

Grimson, A. (2009). Articulaciones cambiantes de clase y etnicidad: una villa miseria de Buenos Aires. En A. Grimson, M. C. Ferraudi Curto \& R. Segura (Comps.), La vida política en los barrios populares de Buenos Aires. Buenos Aires (pp. 221-247). Prometeo.

Halpern, G. (2009). Etnicidad, inmigración y política. Prometeo.

Harvey, D. (2008). El derecho a la ciudad. New Left Review, (53), 23-39.

Holston, J. (2008). Insurgent Citizenship. Disjunctions of Democracy and Modernity in Brazil. Princeton University Press.

Instituto Nacional de Estadísticas y Censos (INDEC). (2010). Censo Nacional de Población, Hogares y Viviendas 2010. https://www.indec.gob.ar/indec/web/Nivel4-Tema-2-41-135

Isin, E. (2009). Citizenship in flux: The figure of the activist citizen. Subjectivity, (29), 367-388.

Jelin, E. (1993). ¿Cómo construir ciudadanía? Una visión desde abajo. European Review of Latin American and Caribbean Studies, (55), 21-37.

Jelin, E. (1996). Las mujeres y la cultura ciudadana en América Latina. Programa Women in the Service of Civil Peace de la División de Cultura. unesco.

Lazar, S. (2008). El Alto, Rebel City: Self and Citizenship in Andean Bolivia. Duke University.

Magliano, M. J. \& Perissinotti, M. V. (2020). La periferia autoconstruida: migraciones, informalidad y segregación urbana en Argentina. EURE, 46(138), 5-23.

Magliano, M. J., Perissinotti, M. V. \& Zenklusen, D. (2015). El origen nacional y la condición migratoria como generadores de conflictos y formas de violencia en un barrio de la periferia urbana de Córdoba. Cuadernos de Antropología Social, (42), 109-124.

Manzano, V. (2007). Del desocupado como actor colectivo a la trama política de la desocupación. Antropología de campos de fuerzas sociales. En M. C. Cravino (Edit.), Resistiendo en los barrios: acción colectiva y movimientos sociales en el Área Metropolitana de Buenos Aires (pp. 101-134). UNGS.

Matossian, B. (2010). Expansión urbana y migración. El caso de los migrantes chilenos en San Carlos de Bariloche como actores destacados en la conformación de barrios populares. Scripta Nova. Revista Electrónica de Geografia y Ciencias Sociales, 14.

Mera, G. S. (2017). Entre el mapa y el croquis: problematizando la segregación espacial de los migrantes paraguayos en la Ciudad de Buenos Aires. Estudios Socioterritoriales. Revista de Geografia, 22, 47-63.

Merklen, D. (2005). Pobres ciudadanos. Las clases populares en la era democrática (Argentina 1983-2003). Gorla.

Mezzadra, S. (2012). Capitalismo, migraciones y luchas sociales. La mirada de la autonomía. Nueva Sociedad, (237), 159-178. 
Mezzadra, S. \& Neilson, B. (2016). La frontera como método o la multiplicación del trabajo. Traficantes de Sueños.

Mitra, S. (2013). Introduction: Citizenship as Cultural Flow - Shifting Paradigms, Hybridization or Plusça Change? En S. Mitra (Ed.), Citizenship as Cultural Flow. Structure, Agency and Power (pp. 1-21). Springer

Mitropoulos, A. (2011). An interview with Angela Mitropoulos. Shift Magazine, 10, 18-21.

Novick, S. (2011). Migraciones en el Cono Sur: políticas, actores y procesos de integración. En B. Feldman Bianco et al. (Comp.), La construcción del sujeto migrante en América Latina: prácticas, representaciones y categorías (109-144). Clacso-Flacso.

Nyers, P. (2015). Migrant citizenships and autonomous mobilities. Migration, Mobility, E Displacement, 1(1), 22-37.

oim-cemla. (2004). Relevamiento y diagnóstico de las asociaciones de la comunidad boliviana en la Argentina.

Ong, A. (2012). Ciudadanía flexible: Las lógicas culturales de la Transnacionalidad. Crítica contemporánea, (2), 1-12.

Papadopoulos, D. \& Tsianos, V. (2013). After citizenship: autonomy of migration, organisational ontology and mobile commons. Citizenship Studies, 17(2), 178-196.

Papadopoulos, D., Stephenson, N. \& Tsianos, V. (2008). Escape Routes: Control and Subversion in the Twenty-First Century. Pluto Press.

Penchaszadeh, A. P. \& Condori Mamani, S. (2017). Ciudadanía migrante en Argentina ¿Hecho o proyecto? Revista Temas de Antropología y Migración, (8), 26-40.

Perissinotti, M. V. (2016). Un lugar donde vivir. Las luchas migrantes por el acceso al espacio urbano en la ciudad de Córdoba (Argentina). REMHU-Revista Interdisciplinar da Mobilidade Humana, 24(47), 59-76.

Prévot Schapira, M. F. (2001). Fragmentación espacial y social: conceptos y realidades. Perfiles Latinoamericanos, 19, 33-56.

Rho, M. G. (2020). De las luchas por una nueva ley de migraciones al paro migrante. Nuevas configuraciones de las luchas migrantes en Argentina. REMHU-Revista Interdisciplinar da Mobilidade Humana, 28(58), 127-145.

Rodrigo, F. (2018). Género y nacionalidad en la cotidianidad de la política. Migrantes bolivianas en un movimiento piquetero de la ciudad de La Plata. Miño y Dávila.

Rodrigo, F. (2019a). Ciudadanía por otros medios: Migración boliviana y violencia en la ciudad de La Plata, Argentina. Dilemas-Revista de Estudos de Conflito e Controle Social, 12, 289-308.

Rodrigo, F. (2019b). Más allá de la vigilancia y el control. Políticas de regularización y migración boliviana en la ciudad de La Plata, Argentina. REMHU-Revista Interdisciplinar da Mobilidade Humana, 27, 179-196. https://doi.org/10.1590/198085852503880005610

Rodrigo, F. (2019c). La nación generizada. Modos femeninos de construir y movilizar la bolivianidad en la periferia de la ciudad de La Plata. Zona Franca. Revista de Estudios de Género, 27, 259-287.

Rodrigo, F. (2021a). Ex-centric citizenship: bolivian migration and violence in Argentina. Citizenship Studies, 25. https://doi.org/10.1080/13621025.2021.1888883

Rodrigo, F. (2021b). Papeles y ciudadanías. Paradojas en el uso de las documentaciones de migrantes bolivianos/as en la Argentina. RUNA, archivo para las ciencias 
del hombre, 42, 333-350.

Sassen, S. (2002). The repositioning of citizenship: Emergent subjects and space for politics. Berkeley Journal of Sociology, 46, 4-25.

Segato, R. L. (2007). La nación y sus otros: raza, etnicidad y diversidad religiosa en tiempos de políticas de la identidad. Prometeo.

Svampa, M. \& Pereyra, S. (2003). Entre la Ruta y el Barrio. La experiencia de las organizaciones piqueteras. Biblos.

Vaccotti, L. (2017). Migraciones e informalidad urbana. Dinámicas contemporáneas de la exclusión y la inclusión en Buenos Aires. EURE, 43(129), 49-70.

Vaccotti, L. (2018). La construcción de un sujeto político: Migrantes y lucha por la vivienda en Buenos Aires. Revista Interdisciplinar da Mobilidade Humana, 26(52), 37-54.

Van Gelder, J. L., Cravino, M. C \& Ostuni, F. (2013). Movilidad social espacial en los asentamientos informales de Buenos Aires. Estudos Urbanos e Regionais, 15(2), 123-137.

Varela Huerta, A. (2015). "Luchas migrantes": un nuevo campo de estudio para la sociología de los disensos. Andamios, 12(28), 145-170.

Vázquez, M. (2005). La nacionalidad migrante entre el género, lo político y la clase: madres, paisanas y piqueteras. Actas del III Congreso Panamericano de Comunicación. Buenos Aires, Universidad de Buenos Aires.

Vichich, N. (2005). El Mercosur y la migración internacional. Expert Group Meeting on International Migration and Development in Latin America and the Caribbean, United Nations Secretariat. México.

Vommaro, G. \& Quirós, J. (2011). “Usted vino por su propia decisión”: Repensar el clientelismo en clave etnográfica. Desacatos, (36), 65-84.

Federico Rodrigo

Argentino. Doctor en ciencias sociales, por la Universidad Nacional de General Sarmiento. Becario Postdoctoral del conicet en el Centro de Investigaciones Sociales. Profesor en la Facultad de Periodismo y Comunicación Social de la Universidad Nacional de La Plata, Argentina. Líneas de investigación: aborda, desde una perspectiva transnacional, las luchas en torno a la ciudadanía de migrantes bolivianos/as en la Argentina. Publicación reciente: Rodrigo, F. (2021). Ex-centric citizenship: Bolivian migration and violence in Argentina. Citizenship Studies, 25. https://doi.org/10.1080/ 13621025.2021 .1888883 